\title{
Evolution of entecavir-resistant hepatitis B virus during entecavir and adefovir dipivoxil combination therapy
}

\author{
YANG WANG $^{1}$, SHUANG LIU $^{1}$, YU CHEN $^{1}$, SUJUN ZHENG $^{1}$, LI ZHOU $^{1}$, TSEN HUA $^{2}$, \\ SHAOFEI SUI ${ }^{2}$, FENGMIN LU ${ }^{3}$ and ZHONGPING DUAN ${ }^{1}$
}

\author{
${ }^{1}$ Artificial Liver Center, Beijing YouAn Hospital, Capital Medical University, Beijing 100069; ${ }^{2}$ R\&D Center Asia Pacific, \\ Qiagen (Shenzhen) Co., Ltd., Shenzhen, Guangdong 518000; ${ }^{3}$ Department of Microbiology and Infectious Disease Center, \\ Peking University Health Science Center, Beijing 100191, P.R. China
}

Received August 27, 2014; Accepted July 14, 2015

DOI: $10.3892 / \mathrm{etm} .2015 .2855$

\begin{abstract}
The emergence of entecavir (ETV) resistance is rare, particularly in a longitudinal study. The aim of the present study was to characterize the evolution of ETV-resistant variants during antiviral therapy using entecavir monotherapy followed by ETV-adefovir dipivoxil (ADV) combination therapy. The study included a prospective cohort of 53 consecutive chronic hepatitis B (CHB) patients. During the 60-month period of ETV therapy, 2 patients exhibited ETV resistance and their medical records were comprehensively reviewed. A total of 25 consecutive serum samples were regularly collected from the 2 patients. All the samples were used to characterize the evolution of the polymerase gene mutations using pyrosequencing. The linkage of the variants was analyzed from 87 reverse transcriptase sequences of 3 selective samples using clone sequencing. The 2 patients presented with viral breakthrough during ETV monotherapy. In patient A, the rtL180M, rtS202G and rtM204V mutant variants were detected using pyrosequencing prior to virological breakthrough. Although the viral load declined following the administration of ADV, the ETV-resistant variants were persistently dominant in the viral populations. In patient B, the rtL180M, rtM204I and rtM204V mutants were present in $\sim 70,30$ and $10 \%$ of the viral populations, respectively, at the time of study entry. In addition, $\mathrm{rtT} 184 \mathrm{~F}$ was present in $\sim 20 \%$ of the viral population during virological breakthrough, at month 24 . The rtL180M, rtT184F and $\mathrm{rtM} 204 \mathrm{~V}$ were predominant during the combination
\end{abstract}

Correspondence to: Professor Fengmin Lu, Department of Microbiology and Infectious Disease Center, Peking University Health Science Center, 38 Xueyuan Road, Beijing 100191, P.R. China E-mail: lu.fengmin@hsc.pku.edu.cn

Professor Zhongping Duan, Artificial Liver Center, Beijing YouAn Hospital, Capital Medical University, 8 Xitoutiao, Beijing 100069, P.R. China

E-mail: zpduan2517@126.com

Key words: hepatitis B virus, entecavir, resistance, pyrosequencing treatment. Clonal analysis further revealed that the $\mathrm{rtS} 202 \mathrm{G}$ or rtT184F was in all cases co-localized with rtL180M and rtM204V in any single virus isolate clone. The results of the present study indicate that the addition of ADV therapy with ETV for treating ETV-resistant mutation may not inhibit the replication of ETV-resistant variants that developed previously in lamivudine-treated CHB patients.

\section{Introduction}

Chronic hepatitis B (CHB) infection is a public health issue that may develop into cirrhosis, hepatic decompensation and hepatocellular carcinoma (HCC) $(1,2)$. The treatment of CHB has changed with the inception of nucleos(t)ide analogues (NAs), including lamivudine (LAM), adefovir dipivoxil (ADV), entecavir (ETV) and telbivudine, which target hepatitis B virus (HBV) reverse transcriptase (RT) activity and inhibit viral replication $(3,4)$. These antiviral effects may improve the virological, biochemical and histological status in the majority of CHB patients. However, the effectiveness of NAs is limited by the emergence of drug-resistant HBV strains, which may cause hepatitis flare and hepatic failure $(1,5,6)$.

Although LAM is not recommended as first-line intervention by current guidelines due to the relatively low genetic barrier to developing resistance, it was the first NA to be marketed and has been widely used as the first-line monotherapy drug for a decade in clinical practice (7-9). ETV, a high genetic barrier antiviral agent, exhibits $>1,500$ times greater potency compared with LAM in vitro $(10,11)$. The development of ETV resistance associated with virological breakthrough in NA-naïve patients has been reported to be rare during 5 years of monotherapy (12). The development of resistance to ETV in HBV requires at least three substitutions in the HBV RT gene, including the LAM-related variants rtL180 and rtM204, in addition to at least one mutation at rtT184, rtS202 or rtM250 (11,13-17).

Understanding the evolution of these drug-resistant variants under different antiviral pressures may aid clinicians to select the correct treatment strategies in a timely manner and to prevent undesirable clinical outcomes. In a previous longitudinal study (16), it was reported that the selection of primary ETV resistance is a two-step process in an NA-naïve patient and 
that the development of resistance is primarily a result of poor compliance with treatment protocols. Furthermore, a combined therapy of ADV plus ETV was considered to be the optimal rescue strategy following previous ETV treatment failure in numerous HBV-infected patients in China, where more potent drugs, such as tenofovir, have not been approved or are not affordable for the majority of the population (18). However, the evolution of ETV resistance during the long-term rescue therapy of ETV plus ADV has not yet been investigated.

To date, direct sequencing following polymerase chain reaction (PCR) amplification is the most commonly used method for detecting a drug-resistant variant; however, this technique is unable to detect variants in $<20 \%$ of the heterogeneous viral population (19). Selecting an alternative approach for clinically monitoring resistant variants is a challenging topic in antiviral research. Pyrosequencing facilitates the detection of small subpopulations of resistant variants, provides quantitative sequence data and enables clinicians to better monitor antiviral therapy (20).

On this basis, pyrosequencing was used in the present study to characterize the evolution of ETV-resistant variants in 2 patients with differing histories of LAM exposure, who received ETV combined with $\mathrm{ADV}$ as a rescue therapy, with the aim of improving CHB treatment.

\section{Materials and methods}

Patients. In total, 53 patients with CHB (21 NAs-naïve patients, 32 LAM-treated patients; age, 16-60 years; 42 male, 12 female) were enrolled in this study between June 2007 and July 2008 in Beijing YouAn Hospital, Capital Medical University (Beijing, China). Once enrolled the patients were treated with ETV once daily. During the 60-month study period, 2 patients (one received $0.5 \mathrm{mg}$ daily, the other received $1.0 \mathrm{mg}$ daily) were identified as ETV-resistant due to virological breakthrough, which was defined as a confirmed increase in the HBV DNA level of $>1 \log _{10}$ copies/ml compared with the nadir HBV DNA level during therapy. The 2 patients received additional administration of ADV at a dose of $10 \mathrm{mg}$ daily as a rescue therapy. Their baseline characteristics are shown in Table I.

During a health screening, patient A (female; age, 43 years) was diagnosed with asymptomatic $\mathrm{CHB}$ infection in the immune-tolerant phase, at an age of 19 years. Between April 1999 and September 1999, at 35 years old, patient A was administered LAM therapy in response to elevated alanine aminotransferase (ALT) levels. Subsequently, partly due to poor medication compliance, patient A ceased LAM therapy without consulting a doctor after the elevated ALT level returned to the normal range. Between May 2004 and October 2004, patient A received interferon- $\alpha 2$ a treatment due to an increase in ALT levels, and subsequently received interferon- $\alpha 2 b$ therapy between October 2004 and September 2006. From July 2007, patient A was recruited in this observational study and received a daily treatment of $0.5 \mathrm{mg}$ ETV in response to abnormal liver function.

During a health screening, patient B (male; age, 49 years) was diagnosed with asymptomatic $\mathrm{CHB}$ infection in the immune-tolerant phase at an age of 40 years. In January 2002, at an age of 44 years, patient B was treated with interferon- $\alpha 1 b$ and LAM in response to elevated ALT levels. After 6 months of the combination therapy, the interferon- $\alpha 1 b$ treatment was discontinued. Due to poor medication compliance, patient B ceased LAM therapy without consulting a doctor in January 2003. In March 2006, patient B resumed LAM therapy due to liver enzyme fluctuations. After 1 year, a YMDD motif mutation was identified in the RT gene of patient B. In March 2007, ADV was added to the therapy of the patient. From July 2007, patient B was recruited into this observational study and received a daily treatment of $1.0 \mathrm{mg}$ ETV in response to non-decreasing HBV DNA levels.

Patients $\mathrm{A}$ and $\mathrm{B}$ were diagnosed with $\mathrm{CHB}$ according to the guidelines of the American Association for the Study of Liver Diseases (7). Histology was characterized according to the Ishak scoring system (21). Neither patient was co-infected with hepatitis D virus, hepatitis $\mathrm{C}$ virus or human immunodeficiency virus. The patients were consecutively monitored every 3 months during the first year of therapy, and every 6 months thereafter, throughout the treatment course. During each follow-up, the patients visited their physicians at the hospital and serum specimens were collected for liver function tests and HBV DNA quantification assays. The HBV DNA and ALT levels of the patients during the 60-month clinical course are shown in Fig. 1. There were no reported issues concerning medication noncompliance. A total of 25 serum samples were obtained from each patient, and any remaining serum samples were stored at $-80^{\circ} \mathrm{C}$ for subsequent research use.

This study was conducted in compliance with the Declaration of Helsinki. The use of the collected serum samples was approved by the Medical Ethics Review Committee of Beijing YouAn Hospital (approval no. LL-2007-002S). Patients A and $\mathrm{B}$ provided written informed consent authorizing access to their medical records and to store the remaining serum specimens for research use.

Measurement of liver function and HBV DNA quantification. ALT and aspartate aminotransferase (AST) levels were measured using kits purchased from Shanghai Kehua Bio-Engineering Co., Ltd. (Shanghai, China) and an Olympus Automatic Biochemical Analyzer (AU5400; Olympus Corporation, Tokyo, Japan) with a cut-off value of $40 \mathrm{IU} / \mathrm{L}$. The levels of viral markers, including hepatitis B surface antigen ( $\mathrm{HBsAg}$ ), hepatitis B e-antigen (HBeAg) and antibody against $\mathrm{HBeAg}$ (anti-HBe) were determined using commercial chemiluminescent immunoassay kits (Beijing Wantai Biological Pharmacy, Beijing, China) on an ARCHITECT i-20000SR automatic chemiluminescence immunoassay analyzer purchased from Abbott Laboratories (Chicago, IL, USA).

The serum HBV DNA level was determined using the Cobas HBV Amplicor Monitor assay (Roche Molecular Diagnostics, Pleasanton, CA, USA) at baseline, then every 6 months during the first year of therapy and annually for the remaining of the treatment. The lower limit of quantification was $50 \mathrm{IU} / \mathrm{ml}$ or 291 copies/ml. From the second year of treatment, the HBV DNA levels were assessed using pyrosequencing (PyroMark Q24 Mdx system; Qiagen GmbH, Hilden, Germany) at 18, 30, 42 and 54 months of follow-up.

$q P C R$. HBV DNA was extracted from $200 \mu 1$ serum samples using QIAamp DNA Blood kit (Qiagen $\mathrm{GmbH}$ ), according to the manufacturer's instructions. Nested PCR was used 
Table I. Baseline characteristics of patients A and B.

\begin{tabular}{|c|c|c|c|}
\hline Parameter & Patient A & Patient B & Normal range \\
\hline Gender $(\mathrm{M} / \mathrm{F})$ & $\mathrm{F}$ & M & - \\
\hline Age (years) & 43 & 49 & - \\
\hline $\operatorname{ALT}(\mathrm{U} / \mathrm{l})$ & 132.40 & 92.1 & $5-40$ \\
\hline $\operatorname{AST}(\mathrm{U} / \mathrm{l})$ & 91.90 & 48.1 & $8-40$ \\
\hline $\operatorname{TBil}(\mu \mathrm{mol} / \mathrm{l})$ & 20.70 & 25.5 & $5-20$ \\
\hline $\operatorname{ALP}(\mathrm{U} / \mathrm{l})$ & 69.10 & 74.5 & $35-115$ \\
\hline BUN (mmol/l) & 3.17 & 4.97 & $2.29-7$ \\
\hline CREA $(\mu \mathrm{mol} / \mathrm{l})$ & 46.00 & 67.00 & $53-106$ \\
\hline $\operatorname{ALB}(g / l)$ & 41.80 & 45.80 & $36-55$ \\
\hline $\mathrm{WBC}\left(10^{9} / 1\right)$ & 4.46 & 5.60 & $4-10$ \\
\hline $\mathrm{Hb}(\mathrm{g} / \mathrm{l})$ & 135 & 144 & $110-160$ \\
\hline $\operatorname{PLT}\left(10^{9} / 1\right)$ & 117 & 128 & $100-300$ \\
\hline Prothrombin duration (sec) & 12.70 & 12.6 & $10.7-14.4$ \\
\hline INR (ratio) & 1.06 & 1.04 & - \\
\hline \multicolumn{4}{|l|}{ CLIA } \\
\hline HBsAg & $>250$ (positive) & $>250$ (positive) & $<0.05$ \\
\hline Anti-HBsAg & 2.06 (negative) & 0.00 (negative) & $<10$ \\
\hline $\mathrm{HBeAg}$ & 0.359 (negative) & 513.846 (positive) & $<1$ \\
\hline Anti-HBeAg & 0.01 (positive) & 15.81 (negative) & $>1$ \\
\hline Anti-HBcAb & 7.83 (positive) & 8.35 (positive) & $<1$ \\
\hline LAM therapy duration (months) & 6 & 22 & - \\
\hline HBV DNA $\left(\log _{10}\right.$ copies/ml) & 7.80 & 5.31 & 2.46 \\
\hline Genotype & $\mathrm{C}$ & $\mathrm{C}$ & - \\
\hline Histology score (inflammation/fibrosis) ${ }^{\mathrm{a}}$ & $15 / 5$ & $13 / 4$ & - \\
\hline
\end{tabular}

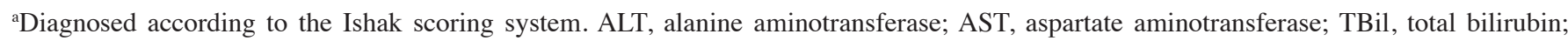
ALP, alkaline phosphatase; BUN, blood urea nitrogen; CREA, serum creatinine; ALB, serum albumin; WBC, white blood cell count; Hb, hemoglobin; PLT, platelet count; INR, international normalized ratio; HBsAg, hepatitis B surface antigen; CLIA, chemiluminescent immunoassay; anti-HBs, antibody against hepatitis B surface antigen; HBeAg, hepatitis B e antigen; anti-HBe, antibody against hepatitis B e antigen; anti-HBc, antibody against hepatitis B c antigen; LAM, lamivudine; HBV, hepatitis B virus.

A

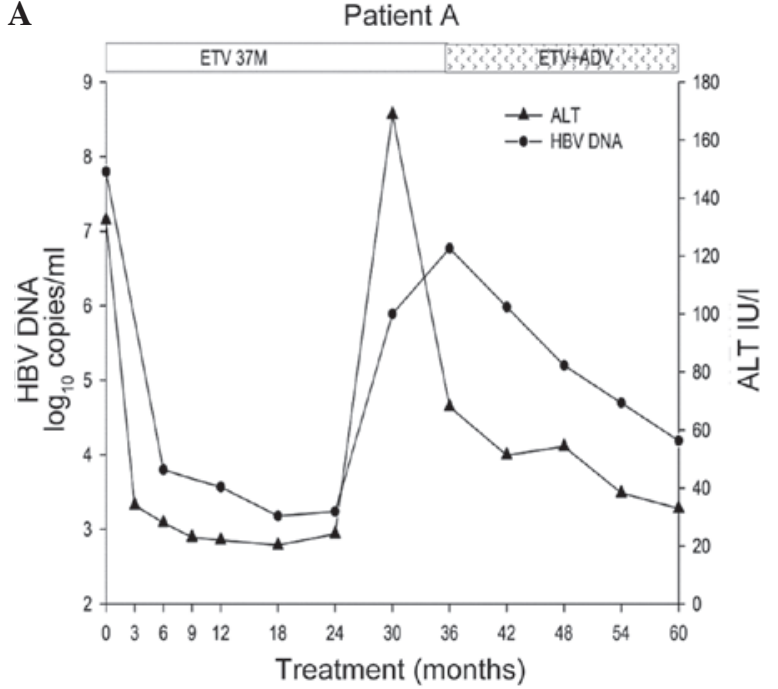

B

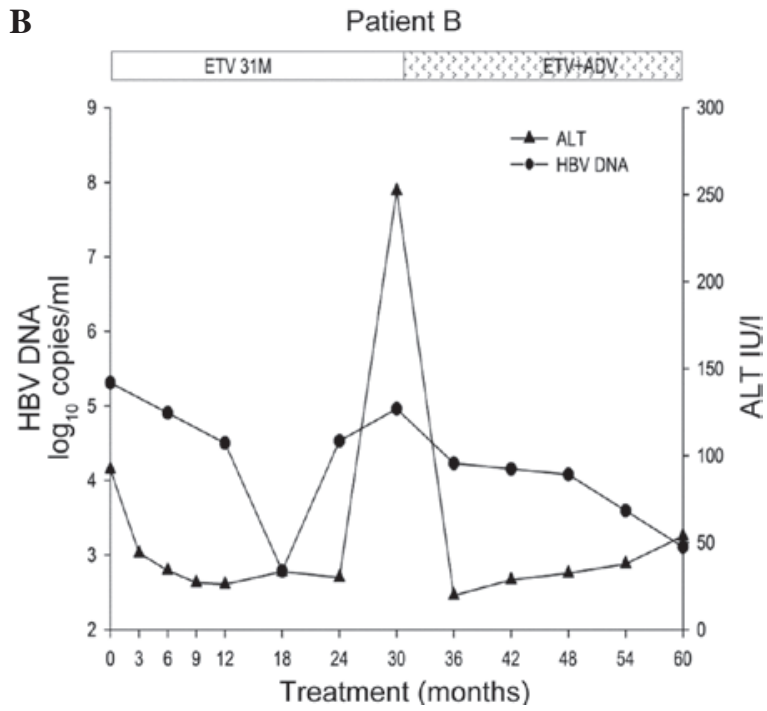

Figure 1. Evolution of the clinical course of (A) patient A and (B) patient B. Therapy duration is indicated by the bars above the graphs. ETV, entecavir; ADV, adefovir dipivoxil; ALT, alanine aminotransferase; HBV, hepatitis B virus. 
to amplify the HBV RT region. PCR was conducted using a ProFlex OCR Veriti 96 thermal cycler purchased from Applied Biosystems (Thermo Fisher Scientific, Inc., Waltham, MA, USA). All primers were synthesized by Sangon Biotech Co., Ltd. (Shanghai, China). A total of $5 \mu 1$ DNA extract was added in the first $25 \mu 1$ reaction using primers $\mathrm{P} 5$ [nt 63-84, 5'-GTGGCTCCAGTTCA(C)GGAACAGT-3'] and P2 (nt 1285-1264, 5'-CTAGGAGTTCCGCAGTATGGAT-3'). PCR conditions were as follows: $94^{\circ} \mathrm{C}$ for $2 \mathrm{~min}$; followed by 30 cycles at $94^{\circ} \mathrm{C}$ for $1 \mathrm{~min}, 63^{\circ} \mathrm{C}$ for $1 \mathrm{~min}$ and $72^{\circ} \mathrm{C}$ for $1.25 \mathrm{~min}$; and $72^{\circ} \mathrm{C}$ for $10 \mathrm{~min}$. The second round of PCR was performed in a $50 \mu 1$ reaction containing $4 \mu \mathrm{l}$ first-round PCR product and primers CN1 (nt 301-319, 5'-TGGCCAAAATTCGCAGTCC-3') and CN2 (nt 1019-1000, 5'-GCAAAGCCCAAAAGACCCAC-3'). PCR conditions were as follows: $94^{\circ} \mathrm{C}$ for $5 \mathrm{~min}$; followed by 30 cycles at $94^{\circ} \mathrm{C}$ for $0.5 \mathrm{~min}, 61^{\circ} \mathrm{C}$ for $40 \mathrm{sec}$ and $72^{\circ} \mathrm{C}$ for $1 \mathrm{~min}$; and $72^{\circ} \mathrm{C}$ for $5 \mathrm{~min}$. Both PCR rounds shared the same final concentration of $\mathrm{MgCl}_{2}(1.5 \mathrm{mM})$, dNTP $(200 \mu \mathrm{M})$, primers (0.8 uM each) and Taq Plus DNA polymerase (50 U/ml) (Dongsheng Biotech Co., Ltd., Guangzhou, China). A 719 bp PCR fragment was purified and sequenced commercially (Invitrogen Life Technologies, Beijing, China) using primers $\mathrm{CN} 1$ and $\mathrm{CN} 2$.

Detection of antiviral-resistant mutations. The pyrosequencing assay was performed according to the standard protocol of the HBV Drug Resistance Test kit (Qiagen Shenzhen Co., Ltd., Shenzhen, China) and the PyroMark Q24 MDx system (Qiagen GmbH). In total, 10 mutation sites were retrospectively analyzed, including $\mathrm{rtL} 169, \mathrm{rtV} 173$, rtL180, rtA181, rtT184, rtA194, rtS202, rtM204, rtN236 and rtM250. These sites are located on the RT domain of HBV DNA polymerase, and were previously reported to be associated with HBV drug resistance $(7,10,14,15,22,23)$.

For the clonal sequencing assay, the PCR products were cloned into the pEASY-Blunt Clone vector (TransGen Biotech Co., Ltd., Beijing, China) according to the manufacturer's instructions. Following transformation into Escherichia coli DH5a competent cells (TransGen Biotech Co., Ltd.), 27-30 colonies per serum sample were selected and the plasmid DNA inserts were sequenced.

\section{Results}

Evolution of ETV-resistant variants associated with the response in patient $A$. Patient A initially received ETV therapy when the serum HBV DNA and ALT levels were $7.80 \log _{10}$ copies/ml and $132.40 \mathrm{IU} / 1$, respectively. The viral load was rapidly reduced to $3.80 \log _{10}$ copies $/ \mathrm{ml}$ after 6 months of treatment, while the ALT level returned to the normal range by month 3 . The HBV DNA level decreased to the lowest level $\left(3.24 \log _{10}\right.$ copies $\left./ \mathrm{ml}\right)$ at month 24 , and then increased to $5.89 \log _{10}$ copies $/ \mathrm{ml}$ at month 30 , indicating virological breakthrough. Simultaneously, the ALT level peaked at $168.8 \mathrm{IU} / 1$ by month 30 , indicating a biochemical breakthrough. The increase in HBV DNA was confirmed at the next re-examination 2 months apart using qPCR $\left(6.63 \log _{10}\right.$ copies $/ \mathrm{ml}$ ). The increase in HBV DNA was confirmed at month 36 using a Cobas HBV Amplicor Monitor assay
(Roche Molecular Diagnostics). Therefore, patient A began a combination therapy of $0.5 \mathrm{mg}$ ETV and $10 \mathrm{mg}$ ADV daily at month 37. After 23 months of combination therapy, the HBV DNA and ALT levels decreased to $4.19 \log _{10}$ copies $/ \mathrm{ml}$ and $32.90 \mathrm{IU} / 1$, respectively (Fig. 1). Compared to the baseline score of 15/5 (inflammation grade/fibrosis stage), the inflammation and fibrosis scores of percutaneous liver biopsy were 6 and 5 after the 60 months of therapy, according to the Ishak classification (21).

The pyrosequencing analysis shown in Fig. 2A is summarized as follows: i) No substitution was detected in the baseline sample; ii) the rtN236T substitution was present in $~ 10 \%$ of the viral population, whereas the wild-type virus was predominantly repressed at month 12 . The $\mathrm{rtN} 236 \mathrm{~T}$ reappeared at month 24 with the viral load fluctuation and was undetectable when outgrowth of the ETV-resistant variants was observed; iii) among the LAM-resistant variants, the rtM204I mutation emerged earlier than rtM204V, at month 18. rtM204I was outcompeted by other mutants, and became undetectable at month 30 , whereas rtM204V and other mutant strains became dominant in the viral population; iv) at month 30 the rtM204V, $\mathrm{rtL180M}$, rtS202G and rtT184S variants were present in $\sim 100$, 100,80 and $15 \%$ of the viral population, respectively, which was accompanied by a virological breakthrough. Prior to the virological breakthrough, the ETV-associated variant, rtS202G, had been detected at month 24 and was present in $\sim 20 \%$ of the viral population; v) after ADV therapy was initiated at month 37, the viral load gradually declined; the rtL180M, rtT184S, rtS202G and $\mathrm{rtM} 204 \mathrm{~V}$ variants were persistently dominant in the viral population during the combination therapy.

A clonal analysis of the samples at month 30 , the point of virological breakthrough, revealed that rtL180M and rtM204 were always co-localized in the same viral strain. At this time, the viral strains of rtL180M + rtM204V + rtS202G, rtL180M + rtM204V + rtT184S and rtL180M + rtM204V + $\mathrm{rtS} 202 \mathrm{G}+\mathrm{rtT} 184 \mathrm{~S}$ were co-existent and represented $83 \%$ $(25 / 30), 10 \%(3 / 30)$ and $7 \%(2 / 30)$ of the viral population, respectively.

Evolution of ETV-resistant variants associated with the response in patient $B$. The ETV treatment reduced the HBV DNA load from $5.31 \log _{10}$ copies $/ \mathrm{ml}$ at baseline to a nadir of $2.79 \log _{10}$ copies $/ \mathrm{ml}$ at month 18 . The ALT levels returned to the normal range at month 6 . Subsequently, the HBV DNA level increased to $4.53 \log _{10}$ copies/ml at month 24 , indicating virological breakthrough, which was supported by the results at month 30. In addition, the ALT levels increased from $29.90 \mathrm{IU} / 1$ at month 24 to $252.3 \mathrm{IU} / 1$ at month 30 , indicating biochemical breakthrough. From month 31, the patient received a combination therapy consisting of $1.0 \mathrm{mg}$ ETV and $10 \mathrm{mg}$ ADV daily. Subsequently, the ALT levels were normalized at month 36, and the HBV DNA level decreased with the extended therapy (Fig. 1). Compared with the baseline score of $13 / 4$ (inflammation grade/fibrosis stage), the inflammation and fibrosis scores of percutaneous liver biopsy after a 60-month therapy were 4 and 3 , respectively, according to the Ishak classification.

The pyrosequencing analysis shown in Fig. 2B may be summarized as follows: i) At baseline, rtL180M, rtM204V and rtM204I were detected in $\sim 30,10$ and $70 \%$ of the viral 
A

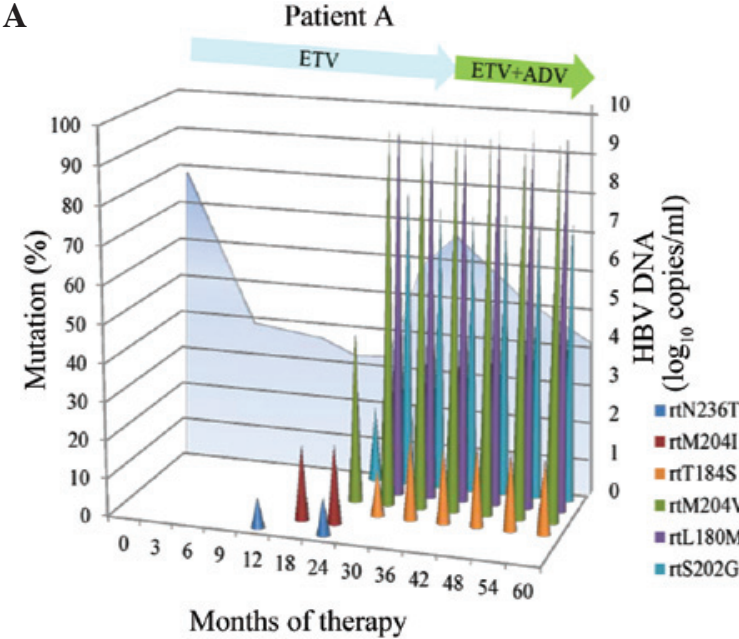

B

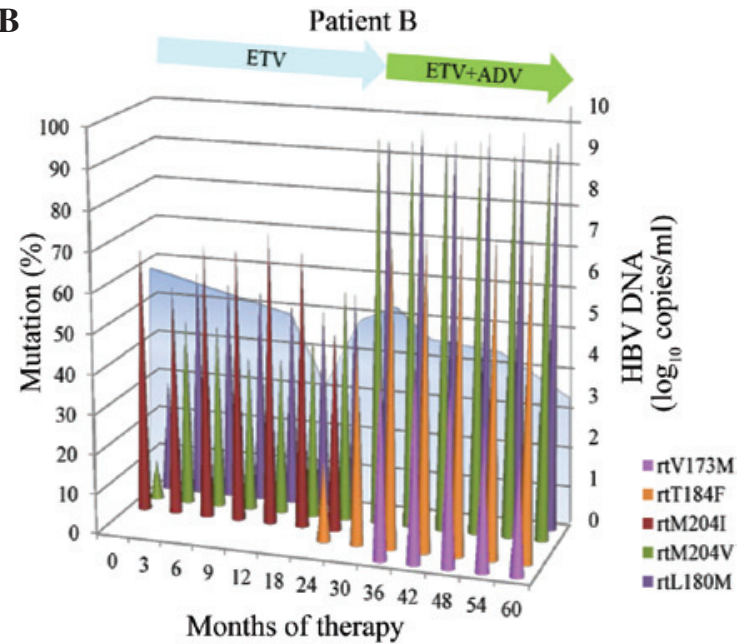

Figure 2. Evolution of resistant variants during ETV and ETV + ADV combination therapy in patients (A) A and (B) B. HBV DNA levels are presented in blue on the back of each graph. ETV, entecavir; ADV, adefovir dipivoxil; HBV, hepatitis B virus.

population, respectively; ii) at month 24 , rtT184F was present in $\sim 20 \%$ of the viral population, within the background of rtL180M and rtM204I/V, which was accompanied by a virological breakthrough; iii) with the outgrowth of rtL180M, rtM204V and rtT184F, rtM204I was gradually outcompeted by other viral mutants and was undetectable in the viral population; iv) following the initiation of ADV treatment at month 31, the quantity of HBV started to decline gradually. rtV173M was detectable in $\sim 50 \%$ of the viral population at month 36 , while rtL180M, rtM204V and rtT184F were persistently dominant in the viral population.

A clonal analysis of the samples at month 24 (a virological breakthrough) and at month 36 (ETV + ADV combination therapy) revealed that rtL180 M and rtM204V were co-localized in the same viral strain. At month 24, the rtL180M + rtM204V, rtM204I and wild-type viral strains co-existed and were present in $17 \%(5 / 30), 47 \%(14 / 30)$ and $37 \%(11 / 30)$ of the viral population, respectively. At month 36, rtT184F emerged and was co-localized with L180M + M204V in the same virus-isolate clone. The rtL180M + rtT184F + rtM204V and rtV173M + rtL180M + rtT184F + rtM204V viral strains represented $33 \%(9 / 27)$ and $67 \%(18 / 27)$ of the viral population, respectively.

\section{Discussion}

In the present longitudinal study, pyrosequencing was used to characterize the evolution of ETV-resistant variants in 2 patients that were previously treated with LAM. The addition of ADV to ongoing ETV treatment for ETV resistance did not appear to suppress the ETV-resistant variants in LAM-treated patients. A clonal analysis of the virological breakthrough samples further revealed that rtT184F or rtS202G were linked with rtL180M and rtM204V and co-localized in the same viral strain. Therefore, the present results suggest that LAM therapy should be cautiously prescribed for NA-naïve patients in the clinical setting.

There is a high genetic barrier to ETV resistance in NA-naïve patients, and only $0.8 \%$ of ETV resistance associated with virological breakthrough was observed during the
5 years of therapy (12). In the present observational cohort study, 2/53 patients presented with ETV resistance associated with virological breakthrough during 5 years of follow-up. Previous LAM exposure may contribute to the higher incidence of ETV resistance. Serum ALT levels may remain normal for a number of weeks following virological breakthrough (10); however, virological and biochemical breakthrough were simultaneously detected at month 30 in patient $\mathrm{A}$. This result does not demonstrate that virological and biochemical breakthrough occurred simultaneously, but indicates that a 6-month interval of follow-up may not be sufficient for certain patients in clinical practice.

Pyrosequencing for resistant variants suggested a two-step process of ETV resistance in LAM-treated patients. In patient A, although previously exposed to LAM for 6 months, no LAM-resistant mutants were detected in the baseline sample, indicated that LAM-resistant mutants were replaced by the wild-type virus after cessation of LAM therapy or that resistant variants had not been selected (24). However, the LAM-resistant variant, rtM204I, was detected after an 18-month period of ETV therapy, which is consistent with previous studies that observed that ETV-resistant variants preceded by LAM-resistant variants $(25,26)$. At month 24 , the resistant variants rtM204V, rtL180M and rtS202G co-existed in the viral population and were co-localized in the same viral strain, while virological breakthrough occurred 6 months later, at month 30 of the therapy. The present results were inconsistent with a previous report, which indicated that a new resistant variant, rtS202G, emerged within the backgrounds of rtM204V and rtL180M, and was accompanied by virological breakthrough (16). This discrepancy may be attributed to the difference of ETV-resistant variants that were proportional in the viral populations.

A previous study reported that ETV resistance occurred more frequently in LAM-treated patients with LAM-resistant variant compared with patients without detectable LAM-resistant variants during ETV monotherapy (27). Prolonged ETV treatment has been associated with an increased risk of acquiring ETV resistance $(28,29)$. Compared with the wild-type virus, the LAM-resistant variant is 8- to 
10-fold less sensitive to ETV $(13,30,31)$. In patient $B$, the baseline sample contained the rtL180M and rtM204I/V variants. At month 24, a new resistant strain that carried all three mutations (rtM204V, rtL180M and rtT184F) emerged, which was accompanied by a virological breakthrough. The results of patient B support the perspective that the LAM-resistant variants were persistently presented during ETV monotherapy $(13,14,32,33)$. This result does not support a previous study, which reported that LAM-resistant variants revert to wild-type HBV during ETV monotherapy (25). The reversion of resistant variants to wild-type $\mathrm{HBV}$ was considered to be a good response to rescue therapy. However, a number of studies have proposed a different explanation for this phenomenon, suggesting that it is only an intermediate step in the selection of novel drug-resistant variants $(25,34,35)$.

There are limited studies concerning the treatment of patients with ETV resistance (13). By using pyrosequencing to detect and quantify the ETV-resistant variants, the present study revealed that the ETV + ADV combination therapy may not suppress the replication of the ETV-resistant strain. This conclusion is suggested by the gradual decline in the serum HBV DNA levels and persistent dominance of ETV-resistant variants in the viral population during the ETV + ADV combination therapy. The histological benefits may be offset by the emergence of resistance of ETV during NA antiviral therapy (36). In the present study, patients underwent liver biopsy at baseline and at month 60 of therapy, and exhibited improved necroinflammation scores; however, neither patient demonstrated a significantly improved fibrosis score. Furthermore, the results indicated that the ETV + ADV combination rescue therapy partially restored the antiviral efficacy of ETV and thus may contribute to the improvement of histology.

Although sensitive pyrosequencing methods were used to analyze consecutive time-point serum samples of up to 60 months of treatment, the present study has some limitations: The small number of patients with ETV resistance, the relatively short duration of ETV + ADV combination therapy, and the testing of only knwon point mutations. It remains a possibility that novel mutations associated with antiviral drug resistance influenced the evolution of resistant variants in the present study. Furthermore, with prolonged treatment, whether the ADV-resistant variant (rtA181V/T or rtN236T) will be selected and linked with the ETV-resistant strain warrants additional investigation.

In conclusion, the additional administration of ADV in combination with ongoing ETV treatment for ETV resistance may not suppress the ETV-resistant variants in patients previously treated with LAM. Although ETV + ADV combination therapy partially restored the antiviral efficacy of ETV, the ETV-resistant variants remained the predominant strains during the 60-month therapy period. The present results suggest that LAM therapy should be cautiously prescribed for NA-naïve patients in clinical practice. The additional benefit of quantifying variants using pyrosequencing may serve as an useful monitoring technique for use in antiviral therapy.

\section{Acknowledgements}

This study was supported by grants from the National Science and Technology Key Project on 'Major Infectious Diseases such as HIV/AIDS, Viral Hepatitis Prevention and Treatment' (nos. 2012ZX10002004-006, 2012ZX10004904-003-001, 2013ZX10002002-006-001 and 2012ZX10002005), High Technical Personnel Training Item in Beijing Health System (no. 2011-3-083), Beijing Municipal Science \& Technology Commission (no. Z131107002213019), Special Scientific Research Fund for Capital Health Development (no. 2011-2018-04) and the Beijing Nova Program (no. Z121107002512056). The authors would like to thank the subjects who participated in this study. The authors are also grateful to Lirong He and Yuping Tang of the R\&D Center Asia Pacific [Qiagen (Shenzhen) Co., Ltd.] for their assistance with the laboratory work and data processing.

\section{References}

1. Peng CY, Chien RN and Liaw YF: Hepatitis B virus-related decompensated liver cirrhosis: benefits of antiviral therapy. J Hepatol 57: 442-450, 2012.

2. Liaw YF and Chu CM: Hepatitis B virus infection. Lancet 373: 582-592, 2009.

3. Buti M: HBeAg-positive chronic hepatitis B: Why do I treat my patients with Nucleos(t)ide analogs? Liver Int 34 (Suppl 1): 108-111, 2014.

4. Viganò $M$, Mangia $G$ and Lampertico P: HBeAg-negative chronic hepatitis B: Why do I treat my my patients with nucleos(t)ide analogs? Liver Int 34 (Suppl 1): 120-126, 2014.

5. Dienstag JL, Goldin RD, Heathcote EJ, et al: Histological outcome during long-term lamivudine therapy. Gastroenterology 124: 105-117, 2003.

6. Zoulim F and Locarnini S: Hepatitis B virus resistance to nucleos(t) ide analogues. Gastroenterology 137: 1593-1608, e1-e2, 2009.

7. Lok AS and McMahon BJ: Chronic hepatitis B: update 2009. Hepatology 50: 661-662, 2009

8. European Association For The Study of The Liver: EASL clinical practice guidelines: Management of chronic hepatitis B virus infection. J Hepatol 57: 167-185, 2012.

9. Liaw YF, Kao JH, Piratvisuth, et al: Asian-Pacific consensus statement on the management of chronic hepatitis B: A 2012 update. Hepatol Int 6: 531-561, 2012.

10. Lok AS, Zoulim F, Locarnini S, et al: Antiviral drug-resistant HBV: standardization of nomenclature and assays and recommendations for management. Hepatology 46: 254-265, 2007.

11. Kobashi H, Fujioka S, Kawaguchi M, et al: Two cases of development of entecavir resistance during entecavir treatment for nucleoside-naive chronic hepatitis B. Hepatol Int 3: 403-410, 2009.

12. Tenney DJ, Rose RE, Baldick CJ, et al: Long-term monitoring shows hepatitis B virus resistance to entecavir in nucleoside-naive patients is rare through 5 years of therapy. Hepatology 49: 1503-1514, 2009.

13. Tenney DJ, Levine SM, Rose RE, et al: Clinical emergence of entecavir-resistant hepatitis B virus requires additional substitutions in virus already resistant to Lamivudine. Antimicrob Agents Chemother 48: 3498-3507, 2004.

14. Villet S, Ollivet A, Pichoud C, et al: Stepwise process for the development of entecavir resistance in a chronic hepatitis B virus infected patient. J Hepatol 46: 531-538, 2007.

15. Lee HW, Kim HJ, Hong SP, et al: Simultaneous emergence of entecavir resistance mutations in a nucleoside-naive chronic hepatitis B patient. Intervirology 55: 380-384, 2012.

16. Lee GH, Inoue M, Toh JK, et al: Two-step evolution of the hepatitis B drug-resistant mutations in a patient who developed primary entecavir resistance. Liver Int 33: 642-646, 2013.

17. Tenney DJ, Rose RE, Baldick CJ, et al: Two-year assessment of entecavir resistance in Lamivudine-refractory hepatitis B virus patients reveals different clinical outcomes depending on the resistance substitutions present. Antimicrob Agents Chemother 51: 902-911, 2007.

18. Ko SY, Kim BK, Kwon SY, et al: Clonal evolution of hepatitis B virus polymerase gene mutations during lamivudine-adefovir combination treatment. World J Gastroenterol 18: 6437-6446, 2012.

19. Degertekin B and Lok AS: Monitoring antiviral resistance in patients receiving nucleos $(\mathrm{t})$ ide analog therapies for hepatitis $\mathrm{B}$ : which method should be used? J Hepatol 48: 892-894, 2008. 
20. Lindström A, Odeberg J and Albert J: Pyrosequencing for detection of lamivudine-resistant hepatitis B virus. J Clin Microbiol 42: 4788-4795, 2004.

21. Goodman ZD: Grading and staging systems for inflammation and fibrosis in chronic liver diseases. J Hepatol 47: 598-607, 2007.

22. Pallier C, Castéra L, Soulier A, et al: Dynamics of hepatitis B virus resistance to lamivudine. J Virol 80: 643-653, 2006.

23. Pallier C, Rodriguez C, Brillet R, Nordmann P, Hézode C and Pawlotsky JM: Complex dynamics of hepatitis B virus resistance to adefovir. Hepatology 49: 50-59, 2009.

24. Chayama K, Suzuki Y, Kobayashi M, et al: Emergence and takeover of YMDD motif mutant hepatitis B virus during long-term lamivudine therapy and re-takeover by wild type after cessation of therapy. Hepatology 27: 1711-1716, 1998.

25. Deng XL, Li QL and Guo JJ: Dynamics of lamivudine-resistant hepatitis B virus strains in patients with entecavir rescue therapy. Virus Genes 47: 1-9, 2013.

26. Guo JJ, Li QL, Shi XF, et al: Dynamics of hepatitis B virus resistance to entecavir in a nucleoside/nucleotide-naive patient. Antiviral Res 81: 180-183, 2009.

27. Lee JH, Cho Y, Lee DH, et al: Prior exposure to lamivudine increases entecavir resistance risk in chronic hepatitis B Patients without detectable lamivudine resistance. Antimicrob Agents Chemother 58: 1730-1737, 2014.

28. Ono A, Suzuki F, Kawamura Y, et al: Long-term continuous entecavir therapy in nucleos(t)ide-naïve chronic hepatitis B patients. J Hepatol 57: 508-514, 2012.
29. Zhao P, Wang C, Huang L, Xu D and Li T: Comparison of rescue strategies in lamivudine-resistant patients with chronic hepatitis B. Antiviral Res 96: 100-104, 2012.

30. Sherman M, Yurdaydin C, Simsek H, et al: Entecavir therapy for lamivudine-refractory chronic hepatitis B: Improved virologic, biochemical and serology outcomes through 96 weeks. Hepatology 48: 99-108, 2008.

31. Colonno RJ, Rose R, Baldick CJ, et al: Entecavir resistance is rare in nucleoside naive patients with hepatitis $B$. Hepatology 44: 1656-1665, 2006.

32. Yim HJ, Hussain M, Liu Y, Wong SN, Fung SK and Lok AS: Evolution of multi-drug resistant hepatitis B virus during sequential therapy. Hepatology 44: 703-712, 2006.

33. Zoulim F: Hepatitis B virus resistance to entecavir in nucleoside naive patients: Does it exist? Hepatology 44: 1404-1407, 2006

34. Ijaz S, Arnold C, Dervisevic S, et al: Dynamics of lamivudine-resistant hepatitis B virus during adefovir monotherapy versus lamivudine plus adefovir combination therapy. J Med Virol 80: 1160-1170, 2008.

35. Villeneuve JP, Durantel D, Durantel S, et al: Selection of a hepatitis B virus strain resistant to adefovir in a liver transplantation patient. J Hepatol 39: 1085-1089, 2003.

36. Chang TT, Liaw YF, Wu SS, et al: Long-term entecavir therapy results in the reversal of fibrosis/cirrhosis and continued histological improvement in patients with chronic hepatitis B. Hepatology (Baltimore, Md.) 52: 886-893, 2010. 